\title{
c-Fos Expression in the Periaqueductal Gray is Induced by Electroacupuncture in the Rat, with Possible Reference to GABAergic Neurons
}

\author{
By \\ Kazutoshi FUSUMADA ${ }^{\mathrm{a}, \mathrm{b}}$, Toshifumi YOKOYAMA ${ }^{\mathrm{b}}$, Takanori MIKI ${ }^{\mathrm{b}}$, \\ Zhi-Yu WANG ${ }^{\text {, }}$ Weiwen YANG ${ }^{\mathrm{b}}$, Nam-Seob LEE ${ }^{\mathrm{c}}$, Yoko ENDO ${ }^{\mathrm{a}}$ \\ and Yoshiki TAKEUCHI ${ }^{\mathrm{b}}$
}

\begin{abstract}
${ }^{a}$ Shikoku Medical College, 62-1 Hamagobanchou, Utazu-cho, Ayauta-gun, Kagawa 769-0205, Japan
${ }^{b}$ Department of Anatomy and Neurobiology, Faculty of Medicine, Kagawa University, 1750-1 Ikenobe, Miki-cho, Kita-gun, Kagawa 761-0793, Japan

'Department of Anatomy, College of Medicine, Konyang University, 26 Nae-dong, Nonsan, Chungnam, 320-711, South Korea
\end{abstract}

- Received for Publication, January 17, $2007-$

Key Words: Periaqueductal gray, Electroacupuncture, c-Fos, GABA, Pain control

\begin{abstract}
Summary: Electroacupuncture (EA) delivered to the acupoint (AP) called Zusanli (ST36) was administered on the bilateral hindlimb. This experiment resulted in strong expression of c-Fos immunoreactivity in the ventrolateral to lateral subdivision throughout the periaqueductal gray (PAG) compared to the non-AP and sham cases. On the other hand, it was of particular interest in the experiment of the AP that strong expression of gamma aminobutylic acid (GABA) frequently showed similar pattern of distribution to that of c-Fos in the PAG. This overlapped pattern of distribution, demonstrated in the present study, suggests that the PAG neurons activated by EA at the AP might play an important role in the descending pain control system involving the GABA since the PAG has special reference to the dorsal horn of the spinal cord and function of pain control.
\end{abstract}

The periaqueductal gray (PAG) refers to the region of the midbrain surrounding the cerebral aqueduct which consists of densely packed small neurons. It has been indicated physiologically that the PAG has a major function of pain control. The PAG is an important site in ascending pain transmission which receives afferent fibers from the spinal cord containing nociceptive neurons and sends fibers to the thalamic nuclei ${ }^{4,13,23)}$. The PAG is also a major component of a descending pain inhibitory system. Activation of this system inhibits nociceptive neurons in the dorsal horn of the spinal $\operatorname{cord}^{1,10,14,35)}$. Additionally, many studies have shown that EA applied to an acupoint (AP) leads to c-Fos expression in various areas of the central nervous system including the spinal $\operatorname{cord}^{20,21,26)}$ and $\mathrm{PAG}^{25,31,36)}$. On the other hand, it has been demonstrated that the EA also induces analge$\mathrm{sia}^{2,25)}$. Stimulation by EA can increase the release of various neuropeptides, such as opioid ${ }^{11,12)}$, endomorphin ${ }^{18,19)}$, enkephalin ${ }^{17)}$, cholecystokinin ${ }^{37)}$ and substance $\mathrm{P}^{16)}$ in the central nervous system. With respect to gamma aminobutyric acid (GABA), one of the major inhibitory neuropeptides, GABA is well known to be contained in the PAG and plays an important role in the descending pain control system $^{7,27,28,30)}$. However, it has not yet been clear whether the PAG neurons activated by EA are GABAergic ones.

Therefore, the present study was performed to elucidate the relationship between c-Fosimmunoreactive (IR) neurons in the PAG induced by EA at the Zusanli (ST36) and GABA-IR neurons using the immunohistochemical technique, and

Address correspondence to: Prof. Yoshiki Takeuchi, Department of Anatomy and Neurobiology, Faculty of Medicine, Kagawa University, 1750-1 Ikenobe, Miki-cho, Kita-gun, Kagawa 761-0793, Japan. e-mail: takeuchi@med.kagawa-u.ac.jp

This work partly supported by a grant-in-aid for Scientific Research from the Ministry of Education, Culture, Sport, Science and Technology of Japan (No. 18591292). 
to establish the GABAergic network of mechanism for output of the PAG.

\section{Materials and Methods}

The present experiments were performed on 33 male Wistar albino rats (SLC, Hamamatsu, Japan), weighing $180-210 \mathrm{~g}$. The animals were housed in separate cages and maintained under standard laboratory conditions $\left(23 \pm 1^{\circ} \mathrm{C}, 12\right.$-h light: 12-h dark cycle, food and water ad libitum). Sham control animals were undergone by the immunohistochemical procedure without any EA $(n=7)$. In experimental animals, the EA was bilaterally applied at the AP of Zusanli (ST36) $(\mathrm{n}=18)$ or at a non-AP $(\mathrm{n}=8)$. Stainless steel needles were inserted to a depth of $5 \mathrm{~mm}$ into the ST36, located between the tibia and the fibula, approximately $5 \mathrm{~mm}$ lateral to the anterior tubercle of the tibia ${ }^{20)}$. The non-AP was located $5 \mathrm{~mm}$ lateral to the midline of the posterior face of the hindlimb. The localization of points was confirmed by measurement of the skin impedance (Lautz, Brazil). Each needle was stimulated using the EA apparatus (SEN-3201, NIHON KOHDEN, JAPAN) for 20 min with electrical pulses at a frequency of $2 \mathrm{~Hz}$ and intensity of $2 \mathrm{~mA}$. The experimental procedures were conducted in accordance with National Institute of Health (NIH) for Care and Use of Laboratory Animals. The Kagawa University Animal Care and Use Committee approved the procedures, and all efforts were made to minimize the number of animals used and their suffering. These EA manipulations were performed under general anesthesia with chloral hydrate $(490 \mathrm{mg} / \mathrm{kg}$, i.p.).

After 1.5-2.0 hours from the onset of the EA stimulation, animals were deeply anaesthetized again and perfused transcardially with $0.02 \mathrm{M}$ phosphate buffered saline (PBS, pH 7.4) followed by fixation with $4 \%$ paraformaldehyde and $0.1 \%$ glutaraldehyde in $0.02 \mathrm{M}$ PBS. The brains were removed, soaked in the same fixative and placed in $20 \%(w / v)$ sucrose in PBS at $4^{\circ} \mathrm{C}$ until they sank. Serial $20 \mu \mathrm{m}$-thick frozen sections through the PAG were cut transversely and processed for Nissl and immunohistochemical staining for c-Fos and GABA using a conventional avidin-biotinimmunoperoxidase technique. Briefly, free-floating sections were rinsed in PBS $(5 \mathrm{~min} \times 3)$, incubated in $5 \%$ skim milk and $0.01 \%$ Tween 20 in PBS for $60 \mathrm{~min}$ at room temperature, and then incubated in c-Fos (1:200 dilution; rabbit polyclonal, Calbiochem, St. Louis, MO, USA) and GABA (1:8000 dilution; mouse monoclonal, Sigma, St. Louis, MO, USA) antibody and kept at $4^{\circ} \mathrm{C}$ overnight. Follow- ing rinsing in PBS $(5 \min \times 3)$, the sections were incubated in biotinylated anti-rabbit or mouse IgG (60 min). Conjugation with HRP labeled avidinbiotin complex (Elite ABC Kit, Vector Lab., USA, (60 $\mathrm{min}$ ) was followed by visualization using a mixture of $0.05 \%$ diaminobenzidine- $0.03 \%$ hydrogen peroxide. Some sections were processed for nickelintensified diaminobenzidine reaction. Stained sections were dehydrated through graded alcohol and xylene, and then coverslipped. Control sections omitting the primary antibody were processed identically and consistently yielded no labeling.

\section{Results}

In Nissl staining, the PAG was definitely confirmed as a thick gray matter which consisted of densely packed small neurons surrounding the cerebral aqueduct. The most rostral region of the PAG is located at the level of the posterior commissure and the most caudal PAG region is located at the level of the dorsal tegmental nucleus. Cytoarchitecturally the PAG was divided into the dorsal, dorsolateral, lateral and ventrolateral subdivisions (including the nucleus of Darkschewitch and dorsal raphe) at the level from the superior colliculus to inferior colliculus in the rat according to the classification by Swanson ${ }^{32)}$ and Medeiros et $a{ }^{25)}$. The PAG was characterized by containing the dorsal raphe nuclei around the ventral portion (Fig. 1).

The c-Fos expression was observed weakly in the whole areas of the PAG except for the ventral portion in the sham control animals (Fig. 2A). Although the expression was increased somewhat in the dorsal subdivision in the experimental animals receiving the EA at the non-AP, the other portions did not show marked changes (Fig. 2B). However, in the experimental animals receiving the EA at the point of Zusanli (ST36), c-Fos expression was characterized by extreme increase in the lateral and ventrolateral subdivisions of the PAG on both side. In particular, this expression showed the tendency of increase at the rostral and middle levels of the PAG (Figs. 2C and 3A, B).

It is of particular interest in the present immunohistochemical study that GABA expression was weak in the sham rats (Fig. 4A), while the expression was much stronger in the AP (Figs. 5A and B) than that in the non-AP animals (Fig. 4B) through the PAG. However, the number of GABAimmunoreactive (IR) neurons showed a tendency to increase at the level of the rostral half of the PAG in the animals receiving the EA at the AP. These IR neurons, oval and triangular in shape, 


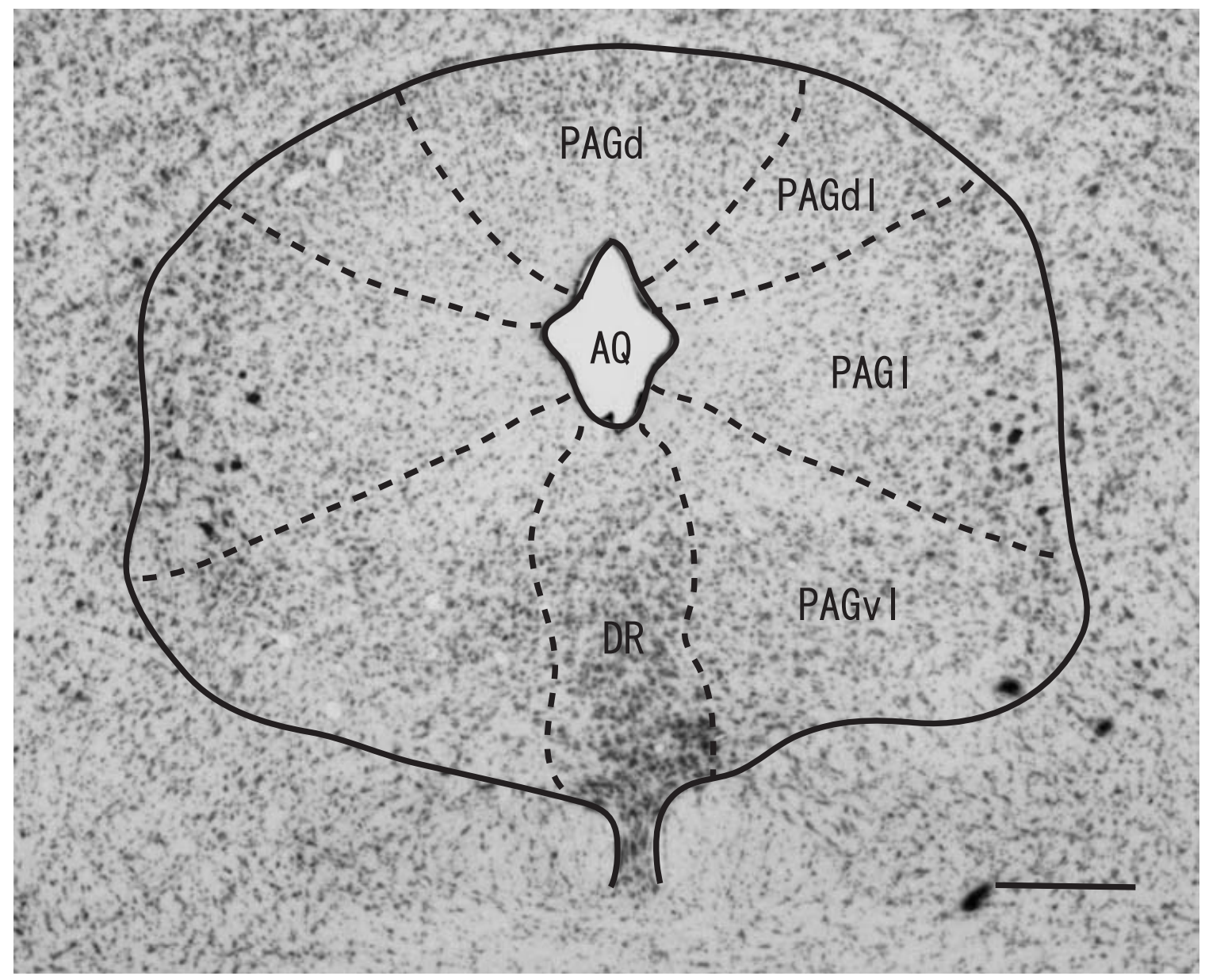

Fig. 1. Nissl staining of the PAG. The PAG is subdivided into the dorsal (d), dorsolateral (dl), lateral (l), ventrolateral (vl) and dosal raphe (DR) subnuclei at the middle level. AQ, cerebral aqueduct; DR, dorsal raphe nucleus; PAGd, dorsal PAG; PAGdl, dorsolateral PAG; PAGl, lateral PAG; PAGvl, ventrolateral PAG. Calibration bar $=500 \mu \mathrm{m}$.

were small in size, and also confined to the ventrolateral to lateral subdivision with some distribution to the dorsal and dorsolateral subdivisions of the PAG. The distribution pattern of GABA-IR neurons was characteristically observed to be overlapped with that of c-Fos-IR neurons.

\section{Discussion}

Many studies have clearly shown that EA applied to an AP leads to c-Fos expression in specific areas of the central nervous system ${ }^{20,21,26)}$. In the midbrain, c-Fos expression is characteristically observed to be present in the ventrolateral to lateral subdivision of the PAG after stimulation of EA at the points of Zusanli (ST36) and non-AP ${ }^{25,36)}$. In the present experiment, EA at the ST36 also induced strong expression of c-Fos immunoreactivity in the same subdivisions throughout the PAG. Recent studies have focused on the ventrolateral to lateral subdivision of the PAG because of having function of pain processing and modulation ${ }^{10,24,29)}$ in contrast to the dorsal subdivision which is a site for vocalization, autonomic regulation, fear, anxiety and lordosis $3,8,9,15)$ and the ventral portion for emotional pinna movements ${ }^{33,34)}$. With respect to the major mechanism of the PAG, it has been established that electrical stimulation around the ventrolateral to lateral subdivision leads inhibition of dorsal horn nociceptive neurons. Initially, it was shown by Liebeskind et al. ${ }^{22)}$ that PAG stimulation inhibits dorsal horn neuronal responses to intraarterial injection of bradykinin Zang et al. ${ }^{35)}$ have shown, using intra- and extra-cellular recording techniques, that stimulation of the PAG preferentially inhibits the response of spinothalamic neurons to $\mathrm{C}$ and $\mathrm{A}$ delta stimulation. The stimulation 


\section{A}

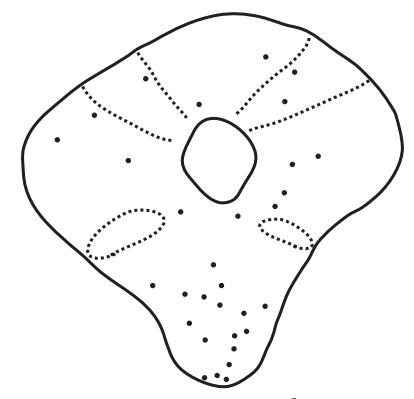

rostral

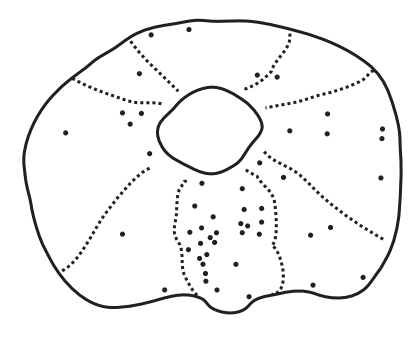

middle
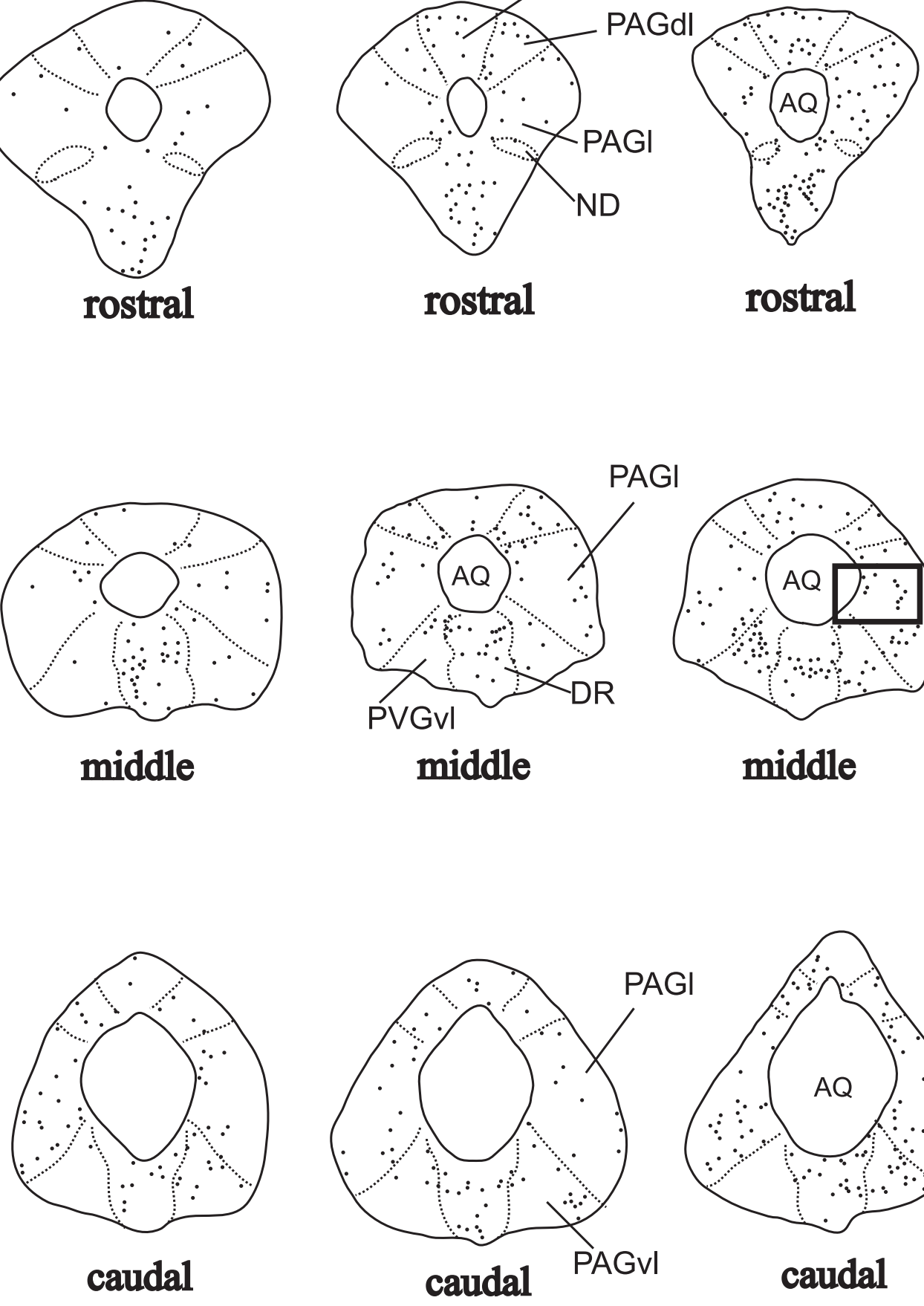

rostral

C

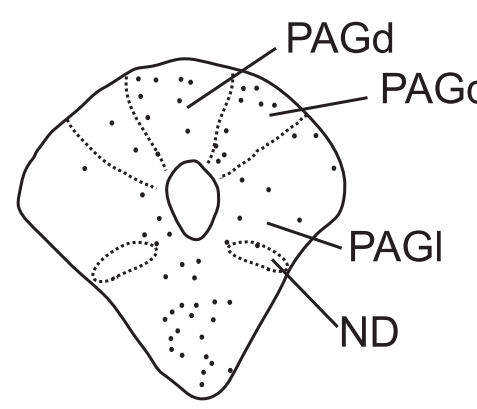

rostral

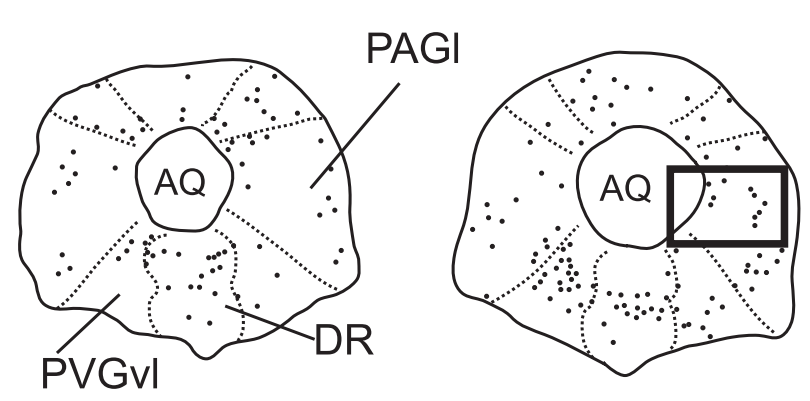

middle

\section{middle}

Fig. 2. Distribution of c-Fos-IR neurons in the PAG in sham (A), non-AP (B) and AP (C) cases at the rostral, middle and caudal levels, respectively. Note that the IR neurons are remarkably increased in the ventrolateral to lateral subdivision throughout the PAG in the AP case. Framed area in $\mathrm{C}$ is shown in Fig. 3A. One dot represents about two IR neurons. AQ, cerebral aqueduct; DR, dorsal raphe nucleus; ND, Darkschewitsch nucleus; PAGd, dorsal PAG; PAGdl, dorsolateral PAG; PAGl, lateral PAG; PAGvl, ventrolateral PAG. 

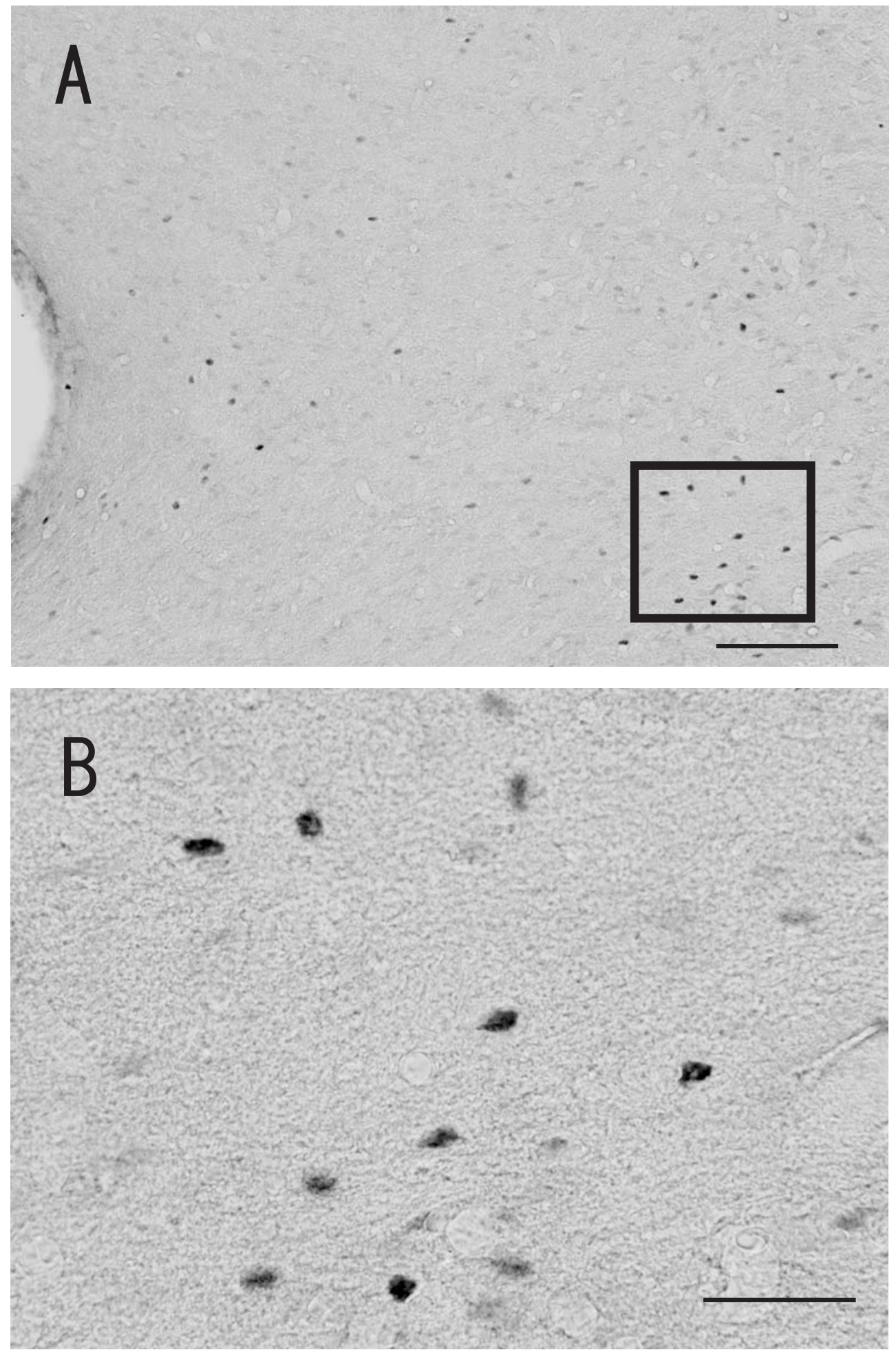

Fig. 3. Photomicrographs of c-Fos-IR neurons in the ventrolateral to lateral subdivision of the PAG at the middle level (A) and its high magnification (B). Framed area in A is enlarged in B. Calibration bars $=50 \mu \mathrm{m}$ in A and $20 \mu \mathrm{m}$ in B. 

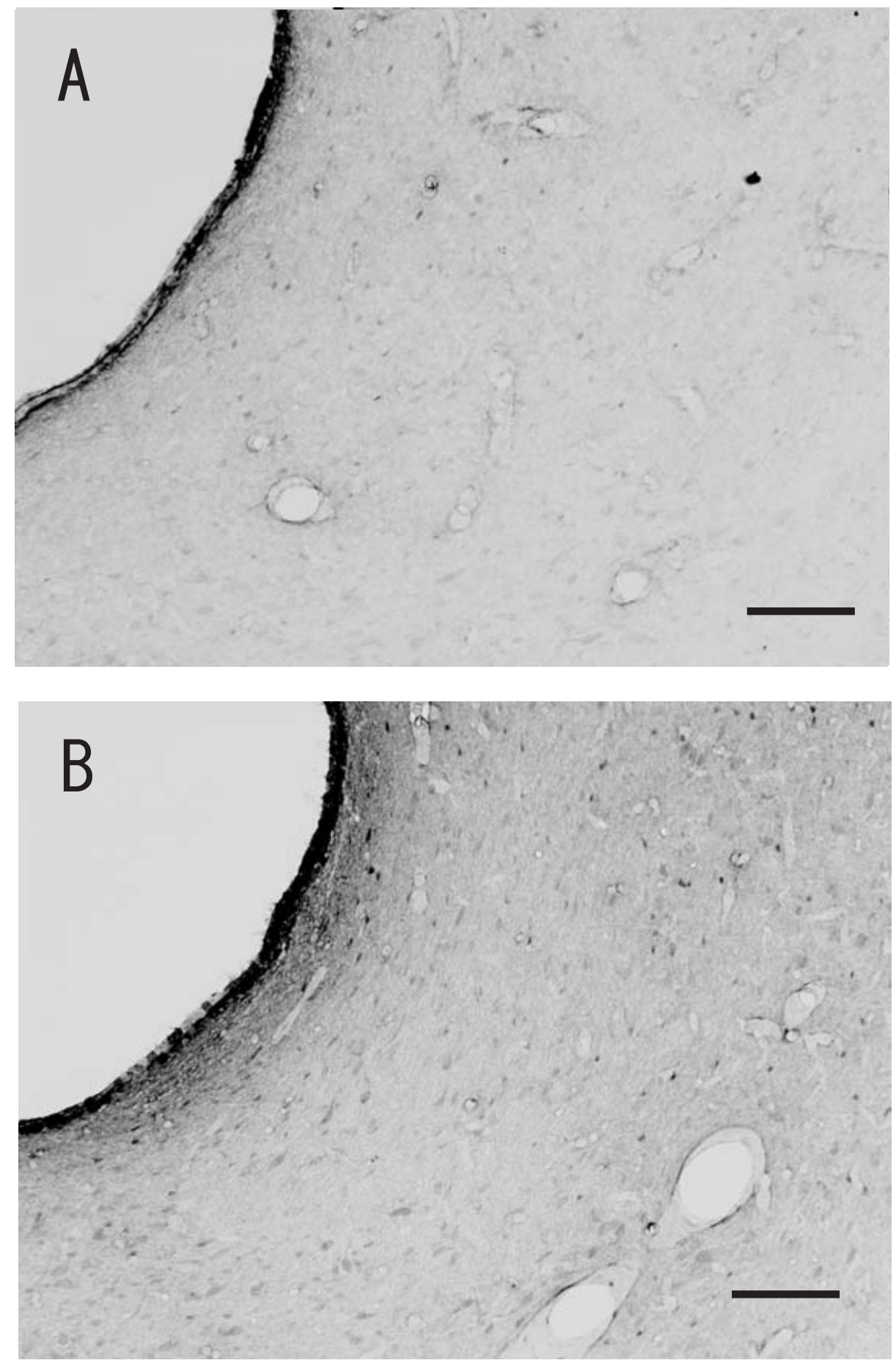

Fig. 4. Photomicrographs of GABA-IR neurons in the ventrolateral to lateral subdivision of the PAG at the middle level in the sham (A) and non-AP (B) cases. Note that expression of GABA immunoreactivity is weak in the sham case, while this expression is strong in the non-AP case. Calibration bars $=100 \mu \mathrm{m}$ in $\mathrm{A}$ and $\mathrm{B}$.

sites within the PAG which inhibit the dorsal horn neurons were distributed mainly into the ventrolateral to lateral subdivision throughout the PAG. The spinal pathway that mediates analgesia, pro- duced by the PAG stimulation, has been established using the lesion experiments and tracer techniques. Early studies by Basbaum and Fields ${ }^{5)}$ established that the dorsolateral funiculus is the 

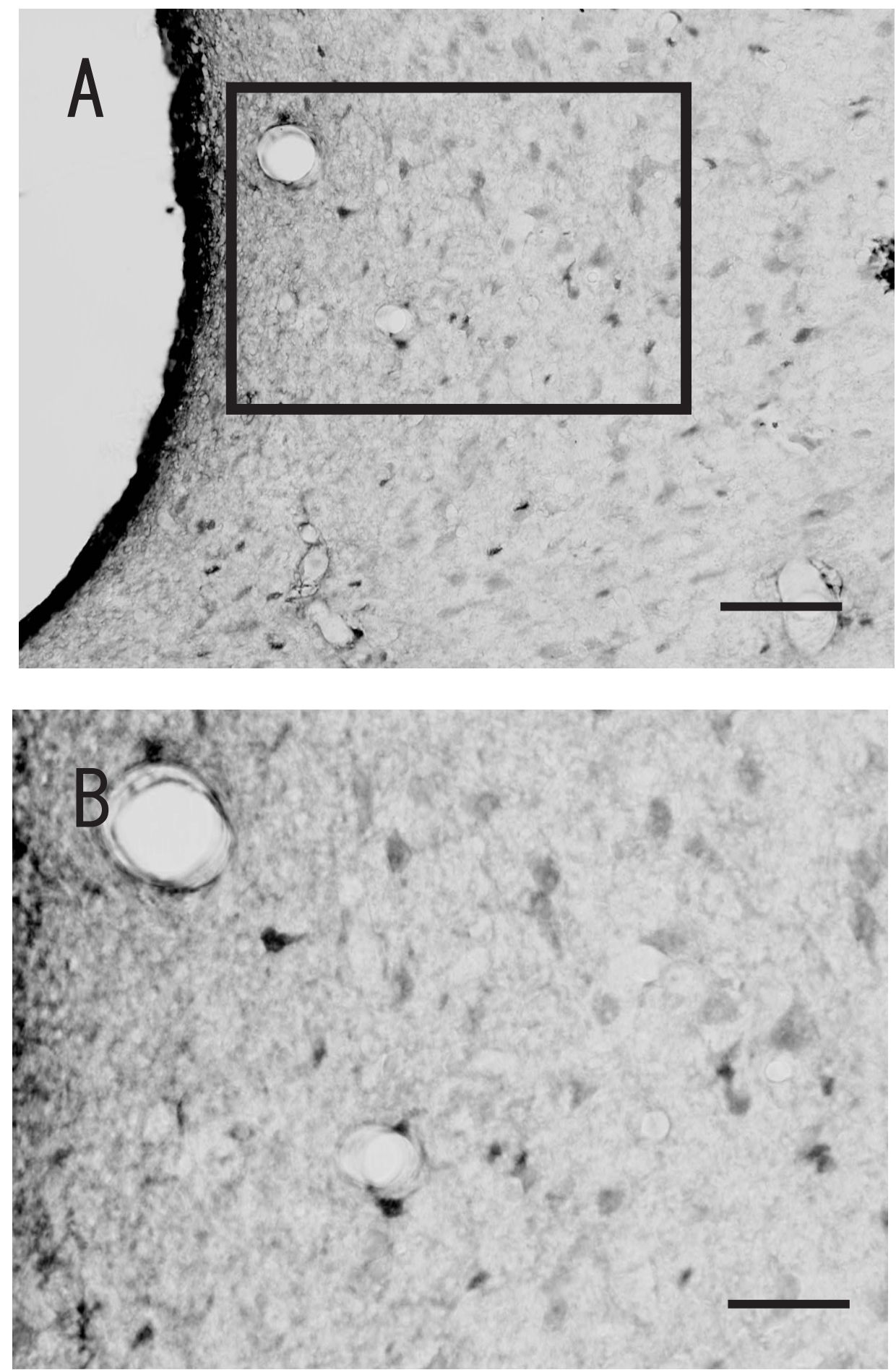

Fig. 5. Photomicrographs of GABA-IR neurons in the ventrolateral to lateral subdivision of the PAG at the middle level in the AP case (A) and its high magnification (B). Note that in this case the expression of GABA immunoreactivity is stronger than that in the non-AP case. Framed area in A is enlarged in B. Calibration bars $=50 \mu \mathrm{m}$ in A and $25 \mu \mathrm{m}$ in $\mathrm{B}$. 
major transmission pathway for the analgesia and its lesion abolishes or significantly attenuates PAGmediated analgesia.

Since there is only sparse direct projection from the PAG to the spinal cord, and since the majority of the axons that travel through the dorsolateral funiculus do not arise from neurons located in the PAG, it was postulated that stimulation-producedanalgesia is mediated through activation of medullary regions ${ }^{5,6}$. Anatomical studies by Reichling and Basbaum ${ }^{27,28)}$ have shown that the medullary regions of the nucleus raphe magnus and paragigantocellularis are the major source of the dorsolateral funiculus axons. In these findings it was described that only $1.5 \%$ of GABA-IR neurons were cells of origin of the PAG-nucleus raphe magnus pathway and very few of the neurons were those of the nucleus raphe magnus-spinal cord pathway in spite of abundant GABA-IR neurons in the PAG. However, in the area including the boundaries of the nucleus raphe magnus and adjacent reticular formation, $15 \%$ of GABA-IR neurons were cells of origin of the nucleus raphe magnus (boundaries)spinal cord pathway. Taken together, it appears to be led that the majority of GABAergic neurons in the PAG and nucleus raphe magnus are interneurons regulating directly or indirectly the activity of the outputs from these areas.

The major intrinsic circuit within the PAG seems to be a tonically active GABAergic network and inhibition of this network is an important mechanism for activation of output of the PAG. It was indicated in the present study that c-Fos expression in the PAG induced by EA at the ST36 shows a highly site-specific distribution pattern and some of c-Fos-IR neurons appeared to be GABA-IR neurons. However, further studies including the double immunohistochemical demonstration should be done to establish the GABAergic network of mechanism for output of the PAG in detail.

\section{Acknowledgements}

We thank Mr. Wakashi Nagata and Mrs. Mizue Fukutomi for their excellent technical assistance.

\section{References}

1) Aimone LD and Gebhart GF. Stimulation-produced spinal inhibition from the midbrain in the rat is mediated by an excitatory amino acid neurotransmitter in the medial medulla. J Neurosci 1986; 6:1803-1813.

2) Akil H, Madden J, Patrick RL and Barchas JD. Stress induced increase in endogeneous opiate peptides: concurrent analgesia and its partial reversal by naloxone. In H.W.
Kosterlitz (Ed.). Opioites and Endogeneous Opioides, Elsevier, Amsterdam, 1976; pp. 63-70.

3) Bandler $R$ and Carrive P. Integrated defence reaction elicited by excitatory amino acid microinjection in the midbrain periaqueductal grey region of the unrestrained cat. Brain Res 1988; 439:95-106.

4) Basbaum AI and Fields HL. Endogeneous pain control mechanisms: Review and hypothesis. Ann Neurol 1978; 4:451-462.

5) Basbaum AI and Fields HL. The origin of descending pathways in the dorsolateral funiculus of the spinal cord of the cat and rat: Further studies on anatomy of pain modulation. J Comp Neurol 1979; 187:513-531.

6) Behbehani MM. Functional characteristics of the midbrain periaqueductal gray. Neurobiol 1995; 46:575-605.

7) Belin MF, Aguera M, Tappaz M, McRae DA, Bobillier P and Pujol JF. GABA-accumulating neurons in the nucleus raphe dorsalis and periaqueductal gray in the rat: a biochemical and radioautographic study. Brain Res 1979; 170:279-297.

8) Carrive P and Bandler R. Control of extracranial and hindlimb blood flow by the midbrain periaqueductal grey of the cat. Exp Brain Res 1991; 84:599-606.

9) Carrive P, Bandler R and Dampney RA. Somatic and autonomic integration in the midbrain of the unanesthetized decerebrate cat: A distinctive pattern evoked by excitation of neurons in the subtentorial portion of the midbrain periaqueductal grey. Brain Res 1989; 483:251-258.

10) Carstens E, Yokota $T$ and Zimmermann M. Inhibition of spinal neuronal responses to noxious skin heating by stimulation of mesencephalic periaqueductal gray in the cat. J Neurophysiol 1979; 42:558-568.

11) Chen $\mathrm{XH}$ and Han JS. Analgesia induced by electroacupuncture of different frequencies is mediated by different types of opioid receptors: another cross-tolerance study. Behav Brain Res 1992; 47:143-149.

12) Chen XH and Han JS. All three types of opioid receptors in the spinal cord are important for $2 / 15 \mathrm{~Hz}$ electroacupuncture analgesia. Eur J Pharmacol 1992; 211:203210.

13) Cliffer KD, Burstein R and Giesler GJJr. Distribution of spinothalamic and spinotelencephalic fibers revealed by anterograde transport of PHA-L in rats. J Neurosci 1991; 11:852-868.

14) Dostrovsky JO, Shah Y and Gray BG. Descending inhibitory influences from periaqueductal gray, nucleus raphe magnus, and adjacent reticular formation. II. Effects on medullary dorsal horn nociceptive and nonnociceptive neurons. J Neurophysiol 1983; 49:948-960.

15) Duggan AW and Morton CR. Periaqueductal stimulation: An association between selective inhibition of dorsal horn neurons and changes in peripheral circulation. Pain 1983; 15:237-248.

16) Go VL and Yaksh TL. Release of substance $P$ from the cat spinal cord. J Physiol 1987; 391:141-167.

17) Han JS, Chen XH, Sun SL, Xu XJ, Yan SC and Terenius JS. Effect of low- and high-frequency TENS on metenkephalin-Arg-Phe and dynorphin A immunoreactivity in human lumbar cerebrospinal fliud. Pain 1991; 47:295-298.

18) Han Z, Jiang YH, Wan Y, Wang Y, Chang JK and Han JS. Endomorphin-1 mediates $2 \mathrm{~Hz}$ but not $100 \mathrm{~Hz}$ electroacupuncture analgesia in the rat. Neurosci Lett 1999; 274:75-78.

19) Huang C, Wang Y, Chang JK and Han JS. Endomorphin and $\mu$-opioid receptors in mouse brain mediate the analgesic effect induced by $2 \mathrm{~Hz}$ but not $100 \mathrm{~Hz}$ electroacupuncture stimulation. Neurosci Lett 2000; 294:159-162. 
20) Lee J-H and Beitz AJ. Electroacupuncture modifies the expression of c-fos in the spinal cord induced by noxious stimulation. Brain Res 1992; 577:80-91.

21) Lee J-H and Beitz AJ. The distribution of brain-stem and spinal cord nuclei associated with difference frequencies of electroacupuncture analgesia. Pain 1993; 52:11-28.

22) Liebeskind JC, Guilbaud G, Besson JM and Oliveras JL. Analgesia from electrical stimulation of the periaqueductal gray matter in the cat: Behavioral observations and inhibitory effects on spinal cord interneurons. Brain Res 1973; 50:441-446.

23) Liu RPC. Laminar origin of spinal projection neurons to the periaqueductal gray of the rat. Brain Res 1983; 264:118-122.

24) Mayer DJ, Wolfe TL, Akil H, Carder B and Liebeskind JC. Analgesia from electrical stimulation in the midbrain of the rat. Science 1971; 174:1351-1354.

25) Medeiros MAD, Canteras NS, Suchecki D and Mello LEAM. Analgesia and c-Fos expression in the periaqueductal gray induced by electroacupuncture at the $\mathrm{Zu}$ sanli point in rats. Brain Res 2003; 973:196-204.

26) Pan B, Castro-Lopes JM and Coimbra A. Chemical senseory deafferenciation abolishes hypothalamic pituitary activation induced by noxious stimulation or electroacupuncture but only decrease that caused by immobilization stress. A c-fos study. Neuroscience 1997; 78:10591068.

27) Reichling DB and Basbaum AI. Contribution of brainstem GABAergic circuitry to descending antinociceptive controls: I GABA-immunoreactive projection neurons in the periaqueductal gray and nucleus raphe magnus. J Comp Neurol 1990; 302:370-377.

28) Reichling DB and Basbaum AI. Contribution of brainstem GABAergic circuitry to descending antinociceptive controls: II. Electron microscopic immunocytochemical evidence of GABAergic control over the projection from the periaqueductal gray to the nucleus raphe magnus in the rat. J Comp Neurol 1990; 302:378-393.
29) Reynolds DV. Surgery in the rat during electrical analgesia by focal brain stimulation. Science 1969; 164:444445 .

30) Sandkuhler J, Willmann E and Fu QG. Blockade of GA$\mathrm{BA}(\mathrm{A})$ receptors in the midbrain periaqueductal gray abolishes nociceptive spinal dorsal horn neuronal activity. Eur J Pharmacol 1989; 160:163-166.

31) Sheng LL, Nishiyama K, Honda M, Yaginuma H and Sugiura Y. Supressive effects of Neiting acupuncture on the toothache: an experimental analysis on Fos expression evoked by tooth pulp stimulation in the trigeminal subnucleus pars caudalis and the periaquductal gray of rats. Neurosci Res 2001; 38:331-339.

32) Swanson LW. Brain maps: structure of the rat brain, Elsevier, Amsterdam, 1992.

33) Takeuhci $Y$, Chen $\mathrm{XH}$, Fukui $\mathrm{Y}$, Itoh $\mathrm{M}$, Miki $\mathrm{T}$ and Miyoshi S. Midbrain tegmentum as an intermediate relay station of the periaqueductal-facial pathway in the cat. Brain Res Bull 1993; 31:57-65.

34) Takeuchi $Y$, Nakano $K$, Uemura $M$, Matsuda $K$, Matsushima R and Mizuno N. Mesencephalic and pontine afferent fiber system to the facial nucleus in the cat: A study using the horseradish peroxidase and silver impregnation techniques. Exp Neurol 1979; 66:330-342.

35) Zang D, Owens CM and Willis WD. Two forms of inhibition of spinothalamic tract neurons produced by stimulation of the periaqueductal gray and cerebral cortex. J Neurophysiol 1991; 65:1567-1579.

36) Zhi-Ling Guo, Ali R Moazzami and John C Longhurst. Electroacupuncture induces c-fos express in the rostral ventrolateral medulla and periaqueductal gray in cat: relation to opioid containing neurons. Brain Res 2004; 1030:103-115.

37) Zhou Y, Sun YH, Shen JM and Han JS. Increased release of immunoreactive CCK-8 by electroacupuncture and enhancement of electroacupuncture analgesia by CCK-B antagonist in rat spinal cord. Neuropeptides 1993; 24:139144. 
